\title{
PRINS Non-Coding RNA Regulates Nucleic Acid-Induced Innate Immune Responses of Human Keratinocytes
}

\author{
Judit Danis ${ }^{1,2 *}$, Anikó Göblös ${ }^{1,2}$, Zsuzsanna Bata-Csörgö ${ }^{1,2}$, Lajos Kemény ${ }^{1,2}$ and \\ Márta Széll/2,3
}

${ }^{1}$ Department of Dermatology and Allergology, University of Szeged, Szeged, Hungary, ${ }^{2}$ MTA-SZTE Dermatological Research Group, Szeged, Hungary, ${ }^{3}$ Department of Medical Genetics, University of Szeged, Szeged, Hungary

Cytosolic DNA fragments are recognized as pathogen- and danger-associated molecular patterns that induce a cascade of innate immune responses. Moreover, excessive cytosolic DNA can enhance chronic inflammation, predominantly by activating inflammasomes, and thereby contributing to the pathogenesis of chronic diseases, such as psoriasis. Psoriasis associated non-protein coding RNA induced by stress (PRINS) is

OPEN ACCESS

Edited by:

Jixin Zhong,

Case Western Reserve University,

United States

Reviewed by:

Ping Chen,

Georgetown University School of Medicine, United States

Shanzhong Gong,

University of Texas at Austin,

United States

Hui Liu,

University of California, San

Francisco, United States

${ }^{*}$ Correspondence:

Judit Danis

danis.judit@med.u-szeged.hu

Specialty section: This article was submitted to Inflammation,

a section of the journal

Frontiers in Immunology

Received: 22 June 2017 Accepted: 14 August 2017 Published: 29 August 2017

Citation: Danis J, Göblös A, Bata-Csörgó Z, Kemény L and Széll M (2017) PRINS Non-Coding RNA Regulates Nucleic Acid-Induced Innate Immune Responses of Human Keratinocytes.

Front. Immunol. 8:1053. doi: 10.3389/fimmu.2017.01053 a long non-coding RNA, which has been shown to be associated with psoriasis susceptibility and cellular stress responses; however, the precise mechanism of its action has not been determined. Here, we provide evidence that, in addition to inflammasome activation, cytosolic DNA induces intracellular inflammatory reactions while decreasing PRINS gene expression. Furthermore, PRINS overexpression can ameliorate the inflammatory-mediator production of keratinocytes induced by cytosolic DNA. Overexpression of PRINS resulted in decreased interleukin-6 (IL-6) and chemokine (C-C motif) ligand 5 (CCL-5) expression and secretion. In silico analysis predicted direct binding sites between PRINS and the mRNAs, which was confirmed by an in vitro binding assay and on cellular level. Our results indicated that PRINS binds directly to the mRNAs of IL-6 and CCL-5 at specific binding sites and eventually destabilizes these mRNAs, leading to a decrease in their expression and secretion of the corresponding proteins. These results may indicate a restrictive role for PRINS in inflammatory processes.

Keywords: PRINS, long non-coding RNA, interleukin-6, CCL-5, keratinocyte, poly(dA:dT)

\section{INTRODUCTION}

The skin provides the first line of defense against a variety of environmental, chemical, and physical stimuli and acts as an active member of the innate immune system. Double-stranded DNA (dsDNA) fragments are known to induce antiviral responses in keratinocytes $(1,2)$ as well as induce inflammasome activation and subsequent interleukin (IL)- $1 \beta$ release in these cells $(3,4)$. Moreover, keratinocytes express a wide range of pattern recognition receptors (PRR) for nucleotide fragments $(5,6)$, mainly implicated in antiviral reactions $(2,6)$. These PRRs are required for immune response in an acute infection and might also lead to the exacerbation of chronic inflammatory inherited multifactorial diseases, such as psoriasis (7). Psoriasis is caused by the interplay of professional immune cells and keratinocytes. Cytosolic nucleotide fragments are highly abundant in psoriatic skin (3), leading to chronic activation of professional immune cells (8), and are thought to be an initiator factor in the disease. In particular, cytosolic nucleotide fragments do not lead to antiviral reaction, but instead activate the AIM2 inflammasome signaling in psoriatic keratinocytes (3), leading to prolonged inflammation. 
In the last decade, genome-scale transcription studies have uncovered non-coding RNAs as previously unrecognized players in the dysregulation of inflammatory reaction in psoriatic skin $(9,10)$. The contribution of both microRNAs (miRNAs) and long non-coding RNAs (lncRNAs) to psoriasis has been extensively studied $(11,12)$. We were first to identify a lncRNA potentially contributing to disease susceptibility: PRINS, the psoriasis susceptibility-related non-coding RNA induced by stress, which is highly expressed in psoriatic non-lesional epidermis compared both to lesional or healthy epidermis (13). The expression of PRINS is modified by a diverse set of cellular stressors, including starvation, ultraviolet B (UVB) irradiation, translation inhibition (14), and hypoxia (15). Moreover, microbial stimuli decreased PRINS expression in macrophages (16) and normal human epidermal keratinocytes (NHEKs) (14), and PRINS was recently shown to interact with chemokine (C-C motif) ligand 5 (CCL-5, also known as RANTES) in kidney epithelial cells (15). CCL-5 is a chemokine attracting leukocytes to the site of inflammation. Under inflammatory conditions, keratinocytes produce large amount of CCL-5 to attract antigen-presenting cells (17) and T-cells into the epidermis (18); moreover, CCL-5 is overexpressed in atopic dermatitis and psoriatic lesions (19). Based on these findings, we aimed to investigate whether the PRINS lncRNA contributes to keratinocyte innate immune responses.

According to our results, PRINS can ameliorate dsDNAinduced keratinocyte immune responses. Bioinformatic analysis of the PRINS lncRNA sequence revealed putative interaction sites for interleukin-6 (IL-6) and CCL-5 mRNAs. Destruction of the putative interacting region resulted in the loss of the ability of PRINS to bind to the IL- 6 mRNA. In addition, our functional studies confirmed the regulatory role of the interaction between PRINS and IL-6 mRNA. Our results suggest a general antiinflammatory function for PRINS and provide insight to the role of high PRINS expression in psoriatic non-lesional skin.

\section{MATERIALS AND METHODS}

\section{Cell Culture and Inflammatory Stimuli}

Normal human epidermal keratinocytes used for the experiments were separated from skin specimens obtained from the Plastic Surgery Unit of our department. Written informed consent was obtained from all investigated individuals. The study was approved by the Human Investigation Review Board of the University of Szeged and complied with the ethical standards of research and in accordance with the Helsinki Declaration.

Epidermis was separated from the dermis by overnight incubation in Dispase (Roche Diagnostics, Manheim, Germany), and keratinocytes were obtained after maceration in $0.25 \%$ trypsin. Cells were grown in $75 \mathrm{~cm}^{2}$ cell-culture flasks and were maintained in keratinocyte serum-free medium, containing EGF and BPE (Gibco Keratinocyte SFM Kit; Life Technologies, Copenhagen, Denmark) and supplemented with 1\% antibiotic/ antimycotic solution (PAA Laboratories $\mathrm{GmBH}$, Pasching, Austria) and $1 \%$ L-glutamine (PAA Laboratories), at $37^{\circ} \mathrm{C}$ in a humidified atmosphere with $5 \% \mathrm{CO}_{2}$. The medium was changed every 2 days.
Third passage keratinocytes were seeded into 6-well plates. Cells were primed in supplement-free medium by addition of $5 \mathrm{ng} / \mathrm{ml}$ tumor necrosis factor- $\alpha$ (TNF- $\alpha$ ) and $5 \mathrm{ng} / \mathrm{ml}$ interferon- $\gamma($ IFN- $\gamma)$. After $24 \mathrm{~h}$, cells were transfected with $0.666 \mu \mathrm{g} / \mathrm{ml}$ polydeoxyadenylic acid-polydeoxythymidylic acid double-stranded homopolymer [poly(dA:dT), Sigma Aldrich, Saint Louis, MO, USA] using the X-tremeGENE 9 DNA transfection reagent (Roche Diagnostics). Cells were harvested $12 \mathrm{~h}$ after poly(dA:dT) transfection.

\section{Gene-Specific Overexpression}

For overexpressing PRINS, the AK022045 cDNA sequence [Biological Resource Center (NBRC) National Institute of Technology and Evaluation, Chiba, Japan] was cloned into a pcDNA3.1(+) vector. The empty pcDNA3.1(+) vector served as a control.

The $\triangle$ PRINS construct was created by replacing the AK022045 region (position 538-622) with the following scrambled sequence: GTGCGTGGCGGAGACGTGGTGGTAGAC C G A A T T G A G G A G G A T C C G A A G G T T A G A C G T AGGCGATCGCCGCTTCGGACGCGGTCGC. The $\triangle$ PRINS sequence was created by GeneArt gene synthesis (Thermo Scientific), and cloned into a pcDNA3.1(+) vector.

Transient transfection of NHEK cells was carried out at approximately $70 \%$ confluency in parallel to cytokine priming, using the X-tremeGENE HP DNA transfection protocol, as described by the manufacturer (Roche Diagnostics). The transfection efficiency was $85 \%$ on average, as determined by the transfection of a GFP reporter construct (Lonza, Basel, Switzerland) and analysis of GFP expression by flow cytometry. The effectiveness of overexpression was investigated with real-time RT-PCR (Figure S1 in Supplementary Material).

\section{In Silico Prediction of Interacting Sites}

Sequence complementarity between PRINS (AK022045) and the mRNA of IL-1 $\alpha$ (M28983.1), IL-1 $\beta$ (NM_000576.2), IL-6 (NM_000600.4), IL-8 (NM_000584.3), TNF- $\alpha$ (NM_000594.3), and CCL-5 (NM_002985.2) was analyzed using two algorithms: RIsearch (20), which uses a simplified nearest-neighbor energy model, and INTARNA (21-23), which calculates the free-energy values of the interaction based on predicted global and local structures of mRNAs. The regions predicted by both programs were considered as putative interaction sites.

\section{RNA Isolation and RT-PCR}

Total RNA was isolated from cells using TRIzol ${ }^{\circledR}$ Reagent (Invitrogen Corp., Carlsbad, CA, USA), following the manufacturer's instructions. Turbo DNA-free Kit (Ambion, Life Technologies) was used for the removal of contaminating DNA. cDNA was synthesized from $1 \mu \mathrm{g}$ total RNA using the iScript cDNA Synthesis Kit (Bio-Rad Laboratories, Hercules, CA, USA). Real-time RT-PCR experiments were carried out with the Universal Probe Library system (Roche Diagnostics) using a C1000 Touch Thermal Cycler (Bio-Rad Laboratories) and the primers listed in Table S1 in Supplementary Material. The expression of each gene was normalized to the 18S rRNA gene. Relative mRNA levels were calculated by the $\Delta \Delta \mathrm{Ct}$ method. 


\section{ELISA}

Cell supernatants were centrifuged $\left(5,000 \mathrm{rpm}, 4 \mathrm{~min}, 4^{\circ} \mathrm{C}\right)$ to pellet cell debris, and the amount of cytokines IL-1 $\alpha$, IL-1 $\beta$, IL-6, IL- 8 , CCL-5, and TNF- $\alpha$ was determined byELISA (IL- $1 \alpha$ DuoSet, IL-1 $\beta$ ELISA Duo Set, and TNF- $\alpha$ Duo Set, R\&D Systems, Minneapolis, MN, USA; Human IL-6 Mini TMB ELISA Development Kit, Human IL-8 TMB ELISA Development Kit, Human RANTES ATBS ELISA Development Kit, PeproTech, Rocky Hill, NJ, USA), according to the manufacturer's instructions.

\section{In Vitro Transcription and In Vitro Binding Assay}

PRINS and $\triangle$ PRINS RNA sequences were produced by in vitro transcription from pcDNA3.1(+) containing the AK022045 or $\triangle$ PRINS cDNA sequence, using Transcript Aid T7 In Vitro Transcription Kit (Thermo Scientific). Products were purified by the GeneJET RNA Purification Kit (Thermo Scientific). Quality and sequence-length analyses were carried out on reducing agarose gel-electrophoresis. The single-stranded RNA products were used in a fluorescent binding assay. The fluorescently labeled RNA sequence 5'6-carboxyfluorescein(6-FAM)/GAAGCUCUAU CUCCCCUCCAGGAGCCCAGCUAUGAACUCCUUCUCC ACAAGCGCCUUCGGUCCAGUUGCCUUCUCCCU GGGGCUGCUCCUGGUGUUGCCUGCUGCCUUCCCU GCC-3', comprising positions 91-205 of the IL-6 (NM_000600.4) mRNA sequence, was produced by Integrated DNA Technologies.

An in vitro binding assay was carried out on a Monolith NT.115 Pico MicroScale Thermophoresis instrument (NanoTemper $\mathrm{GmbH}$, Germany), in nuclease-free water, at $25^{\circ} \mathrm{C}$, with $80 \%$ Laser Power, 10\% LED Power, by 2bind $\mathrm{GmbH}$, Regensburg, Germany. Fluorescence enhancement of the 6-FAM labeled specific truncated IL-6 RNA sequence or a 6-FAM labeled unspecific DNA, as negative control, was measured after addition of PRINS or $\triangle$ PRINS. The concentration of fluorescently labeled molecules was $10 \mathrm{nM}$ constantly, while unlabeled RNA concentration ranged from $126.75 \mathrm{nM}$ to $61.9 \mathrm{pM}$. Initial fluorescence was analyzed for binding curves; the formulation used is displayed in Figure S2 in Supplementary Material.

\section{Statistical Analysis}

Experiments were carried out in triplicate with at least three biological repeats, as indicated in figure legends. For statistical analysis, one-way ANOVA was used to compare more than two groups, and one-tailed, paired $t$-test was used to compare two groups. Statistical analysis was carried out using R software, version 3.2.2., and the significance level was set at $p \leq 0.05$.

\section{RESULTS}

\section{Poly(dA:dT) Treatment of Keratinocytes Induces the Expression of Inflammatory Cytokines while Decreasing the Expression of PRINS}

To study how dsDNA influences NHEKs immune function, we analyzed the expression (Figure 1) and secretion (Figure 2) of several inflammatory cytokines and the expression of PRINS upon poly $(\mathrm{dA}: \mathrm{dT})$ exposure.

We confirmed that poly $(\mathrm{dA}: \mathrm{dT})$ significantly induced the expression and secretion of IL-1 $\beta$ in keratinocytes, as described previously (4). In addition, poly(dA:dT) induced very strong mRNA expression of IL- $1 \alpha$, IL- 6 , IL- 8 , and TNF- $\alpha$ in NHEKs (Figures 1B-F).

To gain pronounced inflammatory reaction in NHEKs, a priming step using TNF- $\alpha$ and IFN- $\gamma$ is often applied before poly $(\mathrm{dA}: \mathrm{dT})$ treatment $(3,4)$; however, the cumulative effect of this treatment on the expression of other cytokines has not been examined thoroughly. When cells were primed for $24 \mathrm{~h}$ before poly $(\mathrm{dA}: \mathrm{dT})$ treatment, IL-1 $\beta$ secretion was enhanced by the combination of TNF- $\alpha$ and IFN- $\gamma$ compared to poly $(\mathrm{dA}: \mathrm{dT})$ treatment alone. In contrast, IL-1 $\beta$ mRNA expression was not affected by priming (Figures 1E and 2D). Similarly, priming had no effect on TNF- $\alpha$ mRNA expression (Figure 1F). However, priming induced significantly higher mRNA expression of the other investigated cytokine genes (Figures 1B-D).

In a manner similar to mRNA expression, poly(dA:dT) transfection induced the secretion of the investigated cytokines, and the priming step significantly enhanced the amount of secreted IL-1 $\alpha$, IL-1 $\beta$, and IL- 8 in the keratinocyte supernatant (Figure 2).

As we and others have demonstrated altered PRINS expression upon exposure to inflammatory molecules $(14,16)$, we examined PRINS expression upon poly $(\mathrm{dA}: \mathrm{dT})$ treatment. Poly(dA:dT) alone or combined with cytokine priming decreased the expression of PRINS in NHEKs (Figure 1A). The expression of PRINS returned to the initial level after $24 \mathrm{~h}$ (data not shown), whereas the expression of inflammatory cytokines declined during this period.

\section{PRINS Overexpression Decreased IL-6 and IL-8 Levels in Keratinocytes}

The change in PRINS expression upon inflammatory stimuli suggests the possible contribution of PRINS to inflammatory responses of NHEK cells. To determine whether PRINS can regulate inflammatory cytokine expression, cells were transfected with a construct for overexpressing PRINS during the combined priming and poly $(\mathrm{dA}: \mathrm{dT})$ treatment. Expression and secretion of IL- 6 and IL- 8 was significantly decreased by PRINS overexpression (Figure 3), whereas mRNA expression and secretion of IL- $1 \alpha$, IL- $1 \beta$, and TNF- $\alpha$ were not affected (data not shown). These results suggest that PRINS does not influence inflammasome activation, but instead influences the regulation of other inflammatory processes.

\section{In Silico Analysis Revealed Putative Interacting Sites between the PRINS IncRNA and the IL-6 mRNA}

The mRNA of the chemokine CCL-5 was previously predicted to interact with PRINS; however, it was not reported whether this interaction affects the stability of the CCL- 5 mRNA (15). Therefore, we measured mRNA expression and secretion of CCL-5 during PRINS overexpression, and found that both decreased (Figure S3 in Supplementary Material) in a manner similar to the changes observed for IL-6 and IL-8. The similarity of these expression 

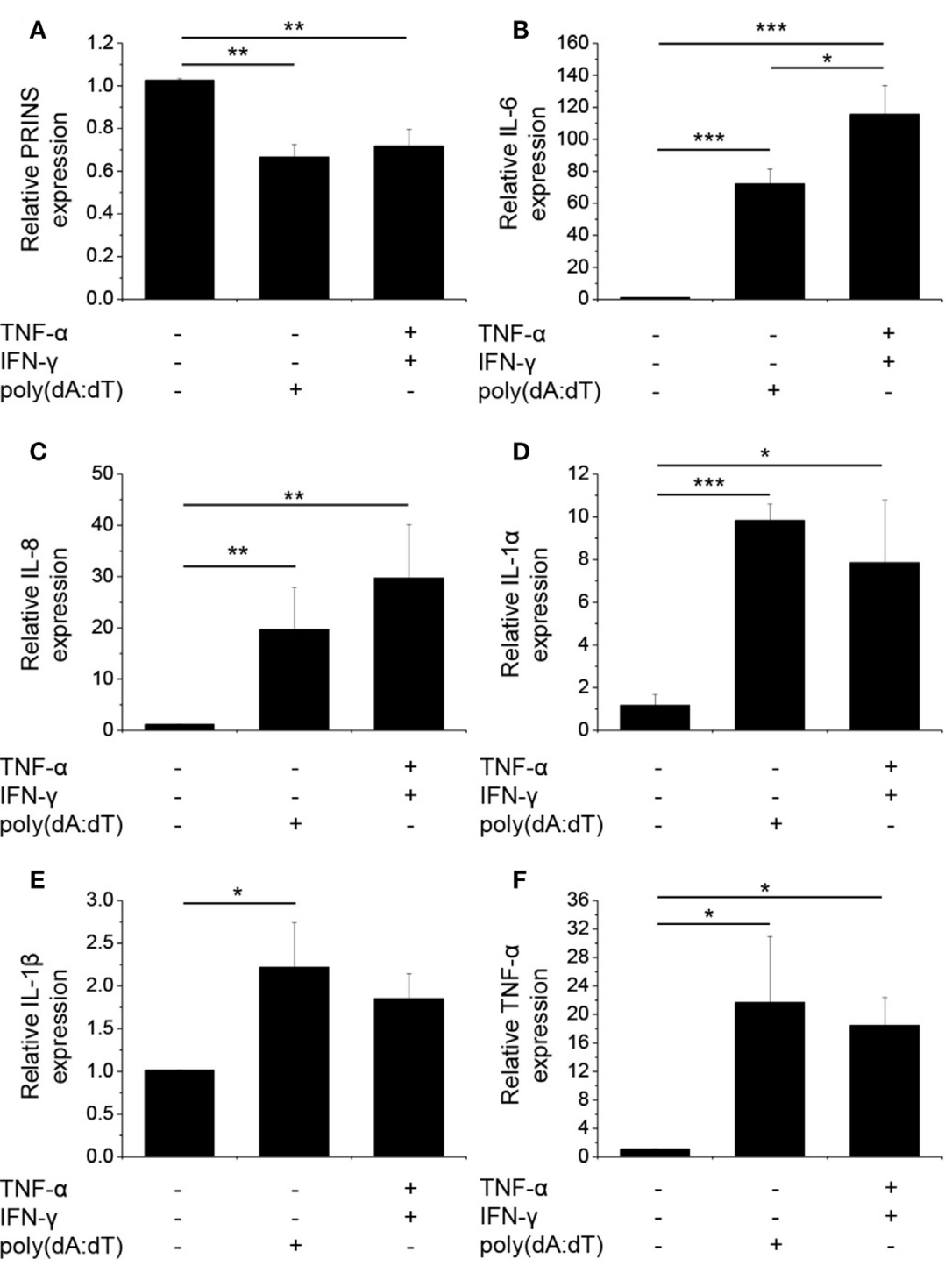

FIGURE 1 | Poly(dA:dT) treatment decreased the expression of PRINS but induced the expression and secretion of cytokines. Normal human epidermal keratinocytes were transfected with poly(dA:dT) for $12 \mathrm{~h}$ with or without priming for $24 \mathrm{~h}$ with $5 \mathrm{ng} / \mathrm{ml}$ tumor necrosis factor- $\alpha$ (TNF)- $\alpha$ and interferon- $\gamma$ (IFN- $\gamma$ ). RNA expression was detected for PRINS (A) and cytokines interleukin-6 (IL-6) (B), IL-8 (C), IL-1 $\alpha$ (D), IL-1 $\beta$ (E), TNF- $\alpha$ (F) by real-time RT-PCR. Data are presented as mean \pm SE of five independent experiments $\left({ }^{*} p \leq 0.05 ;{ }^{* *} p \leq 0.01 ;{ }^{* \star *} p \leq 0.001\right)$.

profiles led us to hypothesize similar mechanism(s) for IL-6, IL-8, and CCL-5 regulation mediated by PRINS.

To predict interactions between PRINS (AK022045) and the mRNAs of IL-6 (NM_00600.4) and IL-8 (NM_000584.3), we performed an in silico analysis using INTARNA $(22,23)$ and RIsearch (20) software. As sequence details of the CCL-5 mRNA and PRINS interaction have not been described in detail (15), we also included the CCL-5 mRNA (NM_002985.2) in the in silico analysis. As a control for the reliability of the prediction analyses, mRNA sequences of cytokines not affected by PRINS overexpression (IL$1 \alpha-M 28983.1$, IL-1 $\beta$-NM_000576.2, TNF- $\alpha-N M \_000594.3$ ) were also included. The regions predicted by both algorithms were considered putative interacting sites. Putative interaction sites were not predicted for the cytokines not affected by PRINS overexpression (IL-1 $\alpha$, IL-1 $\beta$, TNF- $\alpha$ ) in this analysis. PRINS interaction regions were only predicted for the IL- 6 and CCL- 5 mRNAs (Figures 4B,C), two mediators affected by PRINS overexpression. A distance of approximately $200 \mathrm{nt}$ separates the predicted interaction sites in the PRINS sequence, and the corresponding sites occur in the $5^{\prime}$ untranslated region (UTR) of IL- 6 and the $3^{\prime}$ UTR of CCL-5 (Figure 4A). No interaction site was predicted for IL-8.

\section{PRINS Binds to IL-6 mRNA through Direct, Sequence-Specific Interaction}

To validate the predicted interaction site, an in vitro binding experiment was carried out using the PRINS lncRNA and the IL- 6 mRNA. Binding affinity was determined using the 

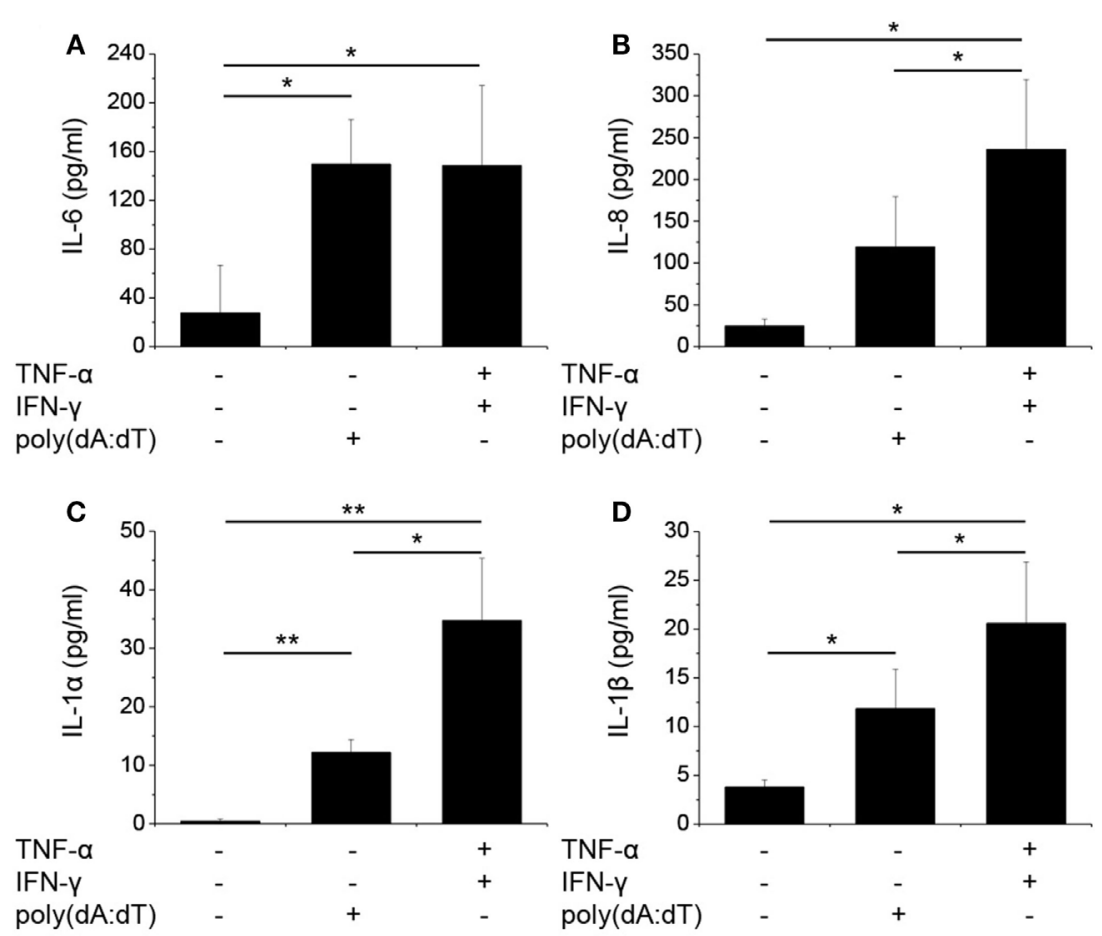

FIGURE 2 | Poly(dA:dT) treatment induced the secretion of cytokines by normal human epidermal keratinocytes (NHEKs). NHEKs were transfected with poly(dA:dT) for $12 \mathrm{~h}$ with or without priming for $24 \mathrm{~h}$ with $5 \mathrm{ng} / \mathrm{ml}$ tumor necrosis factor- $\alpha$ (TNF- $\alpha$ ) and interferon- $\gamma$ (IFN- $\gamma$ ). Secretion of cytokines interleukin-6 (IL-6) (A), IL-8 (B), IL-1 $\alpha$ (C), IL-1 $\beta$ (D) was detected by ELISA from cell supernatant. Data are presented as mean \pm SE of five independent experiments $\left({ }^{\star} p \leq 0.05\right.$; ${ }^{\star \star} p \leq 0.01$; $\left.{ }^{\star * \star} p \leq 0.001\right)$.

single-stranded PRINS RNA and a fluorescently labeled, truncated IL-6 mRNA sequence containing the predicted interacting sequence. The $\Delta$ PRINS sequence, in which the predicted interaction site to IL-6 was replaced by scrambled sequence, was used as a control. As a negative control, a fluorescently labeled DNA sequence with no similarity to either PRINS or IL-6 RNAs was used. While PRINS exhibited a very high binding affinity to the labeled IL-6 mRNA (Figure 5A, Kd = $10.3436 \mathrm{nM}$ ), specific binding for $\triangle$ PRINS and the unspecific labeled DNA could not be detected (Figure 5B). This result confirms in vitro the specificity of the regions predicted in silico (538-622 of PRINS, AK022045 and 92-204 of IL-6 mRNA, NM_000600.4).

\section{PRINS Decreases IL-6 Expression in NHEKs through Sequence-Specific Interaction}

To further validate the functionality of the in silico predicted and in vitro determined IL-6 mRNA interacting region in the PRINS sequence, we performed the overexpression experiments in NHEKs with vectors containing the wild-type PRINS or $\triangle$ PRINS (with scrambled IL-6 binding site) sequences, during the combined priming and poly(dA:dT) treatment. IL-6 expression was not affected by overexpression of $\triangle$ PRINS but was, in contrast, significantly decreased by PRINS overexpression (Figure 6A), and similar tendencies were seen in the amount of secreted IL-6 (Figure 6D). To confirm the specificity of this region in IL-6 regulation, the expression of CCL-5 was also studied. CCL- 5 expression and secretion decreased similarly both in cells overexpressing $\triangle$ PRINS and in cells overexpressing PRINS (Figures 6B,E). IL-8 expression upon PRINS or $\Delta$ PRINS overexpression showed a similar tendency to IL-6 expression, although significant differences could not be detected (Figures 6C,F). These result demonstrated that the binding site in the PRINS sequence is essential and specific for the regulation of IL-6 expression.

\section{DISCUSSION}

Due to large-scale gene-expression studies the number of annotated human non-coding RNAs has increased rapidly (24), but functional roles have been assigned only to a few of them $(25,26)$. PRINS was one of the first lncRNAs described to be dysregulated in a disease, namely in psoriasis (13). PRINS is located in an intron of the KIAA1217 gene on the sense strand (27), and, thus, it can be considered as a long intronic non-coding RNA. The expression of PRINS increases in response to various cellular stressors (14) in $\mathrm{HaCaT}$ cells, as well as to hypoxia in HK-2 cells (15) suggesting that PRINS contributes to the stress response of the cells. Recent reports on PRINS showing decreased expression upon inflammatory stimuli in both NHEKs and macrophages $(14,16)$, and the potential interaction between PRINS and the mRNA of CCL-5 (15) has led us to study its potential contribution to keratinocytes immune responses. 

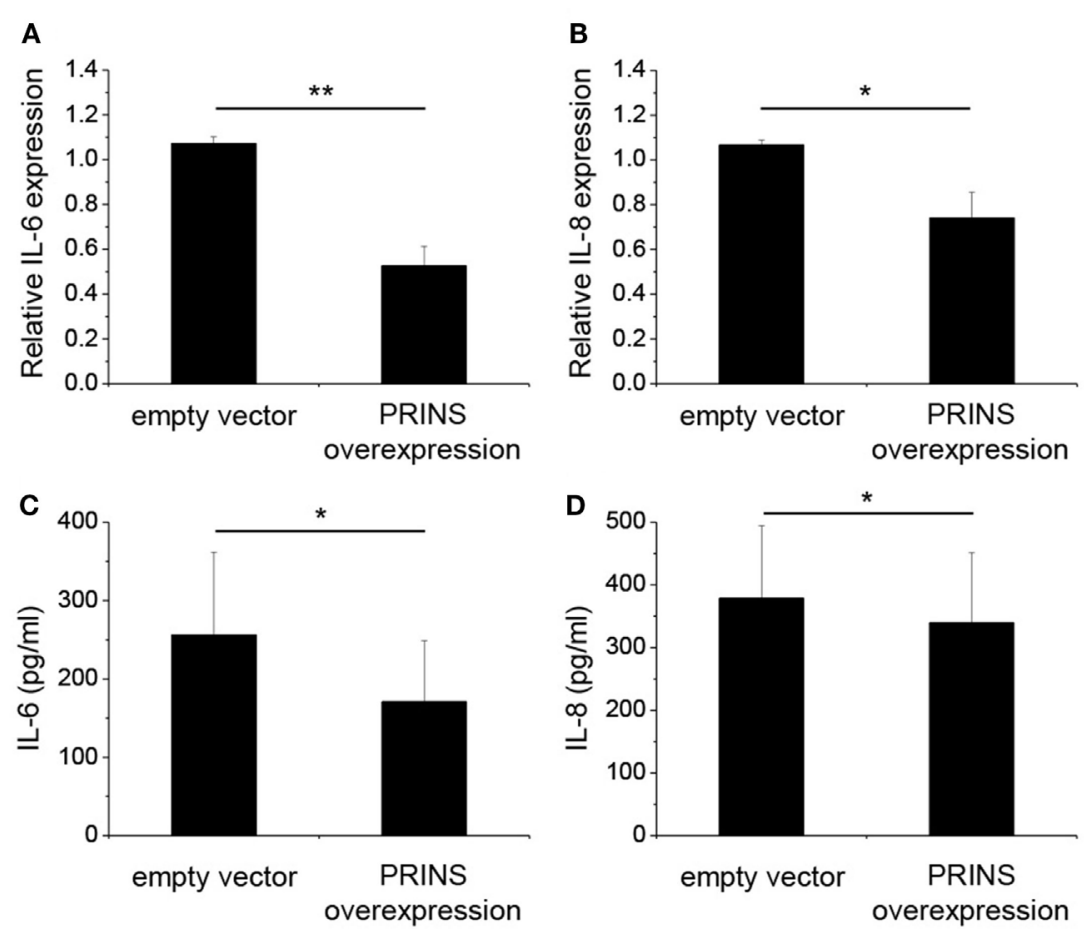

FIGURE 3 | Overexpression of PRINS regulates the expression and secretion of interleukin-6 (IL-6) and IL-8. In parallel to priming with 5 ng/ml tumor necrosis factor$\alpha$ and interferon- $\gamma$, normal human epidermal keratinocytes were transfected with a pcDNA3.1(+) vector containing the PRINS cDNA; an empty pcDNA3.1(+) vector was used as control. After $24 \mathrm{~h}$, cells were transfected with poly(dA:dT). RNA expression of cytokines IL-6 (A) and IL-8 (B) was detected by real-time RT-PCR. Secretion of cytokines IL-6 (C) and IL-8 (D), was detected by ELISA from cell supernatants. Data are presented as mean \pm SE of four independent experiments $\left({ }^{*} p \leq 0.05 ;{ }^{* *} p \leq 0.01\right)$.

To study keratinocyte immune functions, we applied a treatment with the cytosolic DNA-analog poly(dA:dT), which was reported to induce inflammasome activation in these cells $(3,28)$ and induce the expression of several cytokines through a RIG-Idependent mechanism in professional immune cells (29). Before poly(dA:dT) treatment, a priming with the cytokines TNF- $\alpha$ and IFN- $\gamma$ was applied, which were described to be key elements in keratinocyte immune responses (30) and induce a pronounced inflammasome activation (4). Our results are in agreement with recent reports describing inflammasome activation in keratinocytes $(3,4,28)$ and IL- 6 , IL- 8 , and TNF- $\alpha$ induction in canine keratinocytes (6) and human melanocytes (31) upon poly(dA:dT) exposition. Priming induced significantly enhanced mRNA expression of IL-6 and IL-8. In addition, cytokine secretion significantly increased compared to poly (dA:dT) treatment alone. In contrast to cytokine expression, poly(dA:dT) treatment decreased PRINS expression in NHEKs in line with previous reports in NHEKs and macrophages $(14,16)$.

PRINS was recently described to potentially interact with the CCL-5 mRNA (15), but its function remains to be elucidated. In this study, we demonstrate that PRINS overexpression decreases the expression of IL-6, IL-8, and CCL-5 in keratinocytes upon poly(dA:dT) treatment. In addition, decreased IL-6 expression was observed upon PRINS overexpression in UVB-treated samples as well (data not shown). Using in silico analysis, regions in the PRINS sequence were predicted to interact with CCL-5 and IL-6 mRNA. As inhibition of transcription by DNA:IncRNA triplex formation or posttranscriptional destabilization of the mRNA by mRNA-lncRNA duplex formation has been reported (25), we analyzed the sequences and found that the interacting site on IL-6 spans two exons, indicating an mRNA-lncRNA interaction. Moreover, PRINS demonstrated perinuclear localization in keratinocytes (32), making it possible to exert its effect at the posttranscriptional level. The mRNA-lncRNA interaction was validated in vitro: PRINS showed a very high binding affinity $(\mathrm{Kd}=10.3436 \mathrm{nM})$ to IL-6 mRNA, and the destruction of the predicted binding site abolished the ability of PRINS to bind to the IL- 6 mRNA. The binding site of PRINS lies within the $5^{\prime}$ UTR of the IL- 6 mRNA, which is a rare phenomenon. The majority ( $40 \%)$ of lncRNAs bind to the $3^{\prime}$ UTR of their target mRNAs, while only around $5 \%$ of lncRNAs is able to bind to the $5^{\prime}$ UTR of their target (33). The functionality of the mRNA-lncRNA interaction was also observed at the cellular level, as overexpression of $\triangle$ PRINS, in which the IL-6 interacting site is scrambled, did not decrease IL-6 levels. Thus, we demonstrated that PRINS is able to bind the IL- 6 mRNA and this specific interaction destabilizes IL-6 expression and secretion in NHEKs.

Similar to IL-6, PRINS overexpression decreased the expression of IL-8, but no interaction site could be predicted. We hypothesize that this is a secondary effect (34); however, we cannot exclude the possibility of a yet unknown interaction site between PRINS and IL-8 mRNA. 

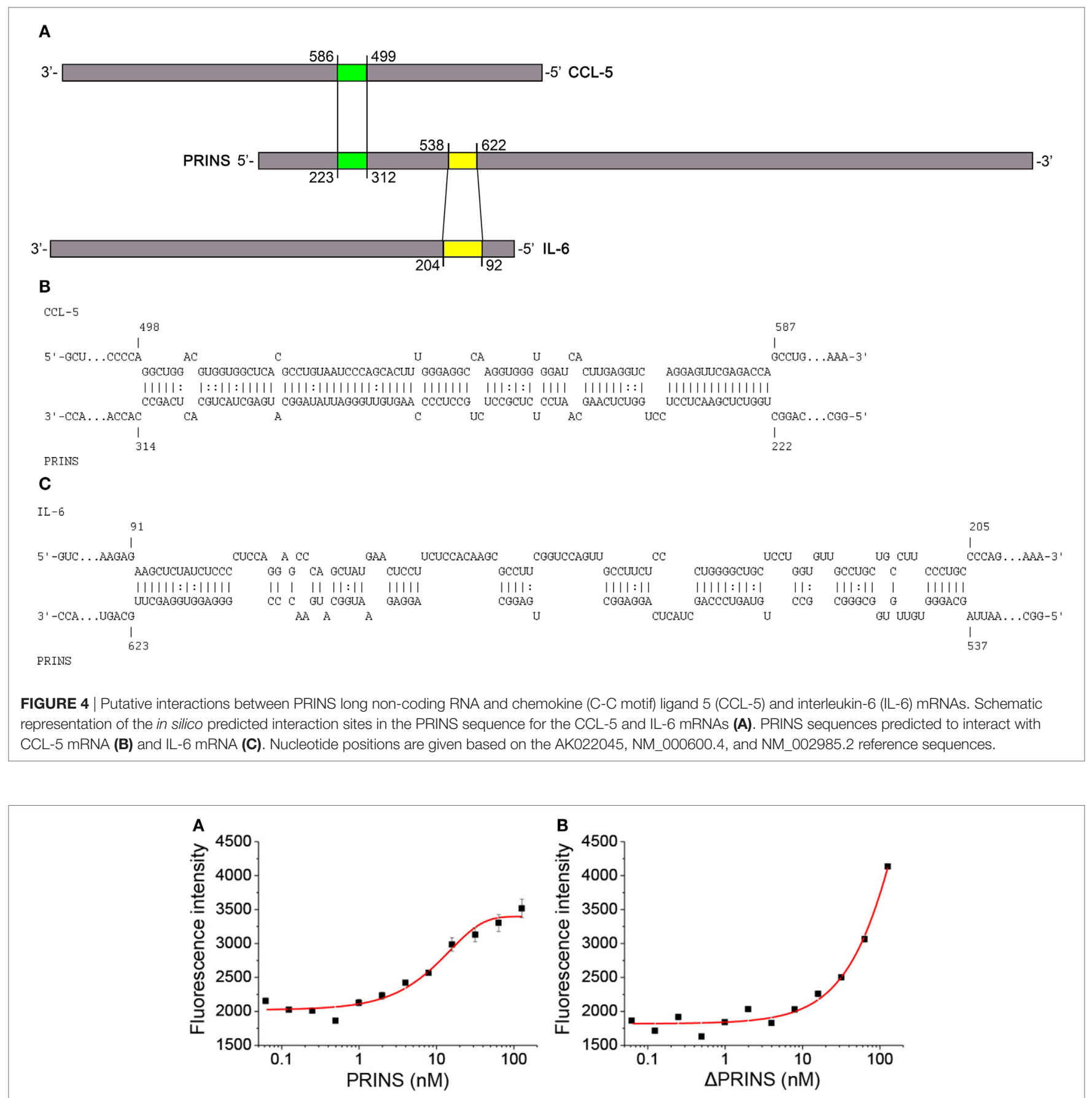

FIGURE 5 | PRINS specifically binds to interleukin-6 (IL-6) mRNA. The binding affinity of PRINS (A) and $\triangle$ PRINS (B) to IL-6 mRNA was determined by analyzing the initial fluorescence enhancement caused by specific binding. The concentration of labeled IL-6 mRNA was constant (10 nM), while the concentration of PRINS and $\triangle$ PRINS varied from $62 \mathrm{pM}$ to $127 \mathrm{nM}$.

Many lncRNAs which were reported to regulate mRNA expression were found to act as primary transcripts for miRNAs (35). We have found no evidence that PRINS is cleaved to smaller miRNA precursors, but the IL-6 binding site of PRINS is $96 \%$ similar to the miR5585, which is predicted by TargetMiner to have IL-6 mRNA as a target (36). miR5585 is located on Chromosome 7, whereas PRINS is located on Chromosome 10, so their high similarity and overlapping functions might originate from gene duplication.

Like many other non-coding RNAs, PRINS seems to have multiple cellular functions (37). It binds to nucleophosmin, a chaperon protein, and facilitates its transition from the nucleolus to the nucleoplasm upon UVB irradiation (32). Additionally, our recent results suggest that PRINS is involved with inflammation 

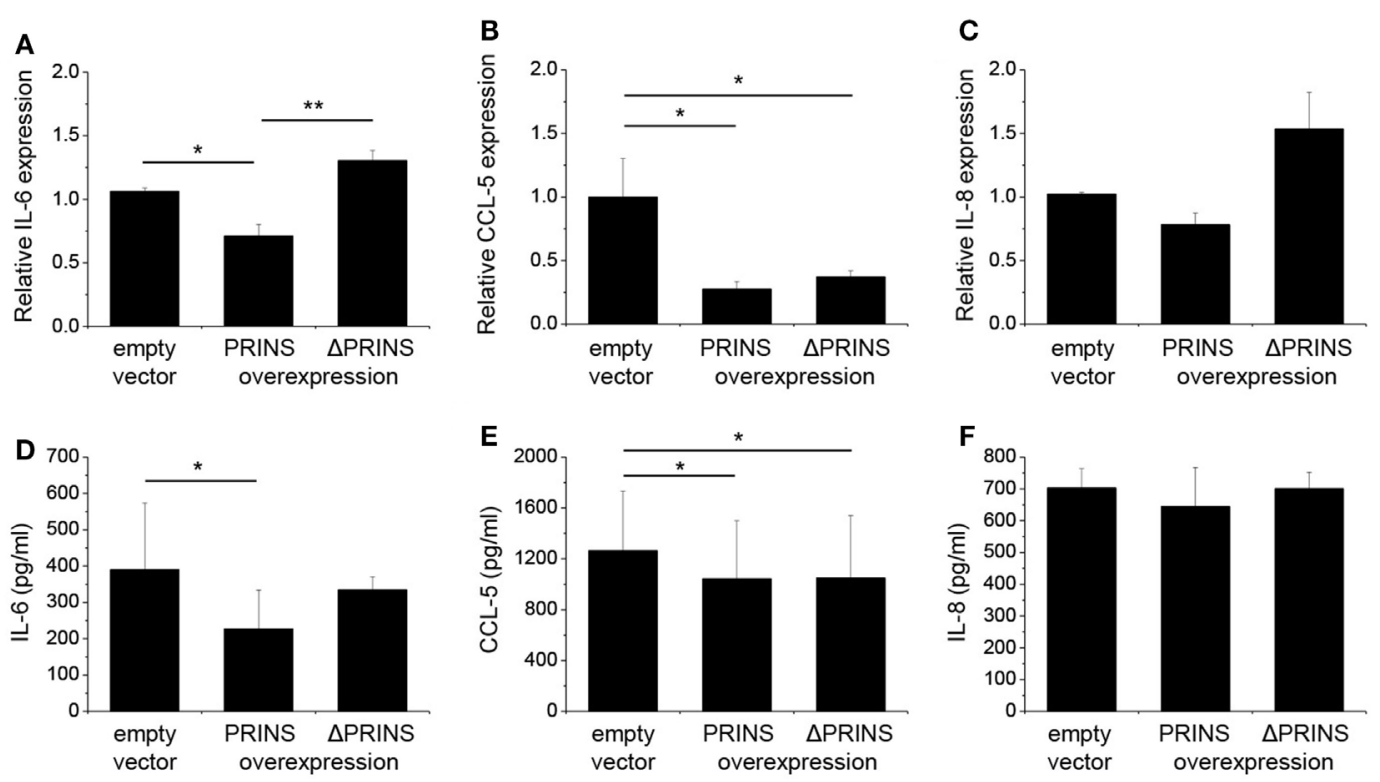

FIGURE 6 | The 538-622 nt region of PRINS is required for the regulation of interleukin-6 (IL-6) expression. In parallel to priming with 5 ng/ml tumor necrosis factor- $\alpha$ and interferon- $\gamma$, normal human epidermal keratinocytes were transfected with a pcDNA3.1(+) vector containing the PRINS cDNA or the $\Delta$ PRINS cDNA (containing a scrambled version of the IL-6 binding site at positions 538-622 nt); the empty pcDNA3.1(+) vector served as control, after 24 hours cells were transfected by poly(dA:dT). Expression of cytokines IL-6 (A), CCL-5 (B), and IL-8 (C) was detected by real-time RT-PCR. Secretion of cytokines IL-6 (D), CCL-5 (E), and IL-8 (F) was detected by ELISA from cell supernatants. Nucleotide positions based on the AK022045 reference sequence. Data are presented as mean \pm SE of three independent experiments $\left({ }^{*} p \leq 0.05 ;{ }^{* *} p \leq 0.01\right)$.

by inhibiting cytokine expression. Based on our current and previous findings (13-15), we hypothesize that elevated PRINS expression in psoriatic uninvolved epidermis may contribute to downregulation of the inflammatory functions in psoriatic keratinocytes and maintenance of normal phenotype.

Our studies were performed using a non-professional immune cell type, keratinocytes; however, the same mechanisms might be also relevant in professional immune cells. Further studies on the same PRINS-related cellular events upon additional stressors such as UVB or microbial components may widen our knowledge on the cellular functions of PRINS and, in general, about lncRNAs.

\section{AUTHOR CONTRIBUTIONS}

JD and MS designed the study, JD and AG prepared laboratory work and analyzed the data. DJ, AG, ZB-C, LK, and MS interpreted

\section{REFERENCES}

1. Luff JA, Yuan H, Kennedy D, Schlegel R, Felsburg P, Moore PF. Keratinocyte antiviral response to poly(dA:dT) stimulation and papillomavirus infection in a canine model of X-linked severe combined immunodeficiency. PLoS One (2014) 9:e102033. doi:10.1371/journal.pone.0102033

2. Suspène R, Mussil B, Laude H, Caval V, Berry N, Bouzidi MS, et al. Selfcytoplasmic DNA upregulates the mutator enzyme APOBEC3A leading to chromosomal DNA damage. Nucleic Acids Res (2017) 45:3231-41. doi:10.1093/nar/gkx001

3. Dombrowski Y, Peric M, Koglin S, Kammerbauer C, Göss C, Anz D, et al. Cytosolic DNA triggers inflammasome activation in keratinocytes in the data and drafted the manuscript. All authors approved the final version.

\section{FUNDING}

This work was supported by the National Research Development and Innovation Office [K105985 to MS, K111885 to ZB-C and GINOP-2.3.2-15-2016-00015]. JD was supported by the NTP-NFTÖ-17 project by the Hungarian Ministry of Human Capacities.

\section{SUPPLEMENTARY MATERIAL}

The Supplementary Material for this article can be found online at http://journal.frontiersin.org/article/10.3389/fimmu.2017.01053/ full\#supplementary-material.

psoriatic lesions. Sci Transl Med (2011) 3:82ra38. doi:10.1126/scitranslmed. 3002001

4. Göblös A, Danis J, Vas K, Bata-Csörgő Z, Kemény L, Széll M. Keratinocytes express functional CARD18, a negative regulator of inflammasome activation, and its altered expression in psoriasis may contribute to disease pathogenesis. Mol Immunol (2016) 73:10-8. doi:10.1016/j.molimm.2016.03.009

5. Prens EP, Kant M, van Dijk G, van der Wel LI, Mourits S, van der Fits L. IFN-alpha enhances poly-IC responses in human keratinocytes by inducing expression of cytosolic innate RNA receptors: relevance for psoriasis. J Invest Dermatol (2008) 128:932-8. doi:10.1038/sj.jid.5701087

6. Luff JA, Yuan H, Suter MM, Müller EJ, Schlegel R, Moore PF. Canine keratinocytes upregulate type I interferons and proinflammatory cytokines in response 
to poly(dA: dT) but not to canine papillomavirus. Vet Immunol Immunopathol (2013) 153:177-86. doi:10.1016/j.vetimm.2013.02.001

7. Lowes MA, Suárez-Fariñas M, Krueger JG. Immunology of psoriasis. Annu Rev Immunol (2014) 32:227-55. doi:10.1146/annurev-immunol-032713-120225

8. Lande R, Gregorio J, Facchinetti V, Chatterjee B, Wang Y-H, Homey B, et al. Plasmacytoid dendritic cells sense self-DNA coupled with antimicrobial peptide. Nature (2007) 449:564-9. doi:10.1038/nature06116

9. Tsoi LC, Iyer MK, Stuart PE, Swindell WR, Gudjonsson JE, Tejasvi T, et al. Analysis of long non-coding RNAs highlights tissue-specific expression patterns and epigenetic profiles in normal and psoriatic skin. Genome Biol (2015) 16:24. doi:10.1186/s13059-014-0570-4

10. Li B, Tsoi LC, Swindell WR, Gudjonsson JE, Tejasvi T, Johnston A, et al. Transcriptome analysis of psoriasis in a large case-control sample: RNAseq provides insights into disease mechanisms. J Invest Dermatol (2014) 134:1828-38. doi:10.1038/jid.2014.28

11. Sonkoly E, Wei T, Janson PCJ, Sääf A, Lundeberg L, Tengvall-Linder M, et al. MicroRNAs: novel regulators involved in the pathogenesis of psoriasis? PLoS One (2007) 2:1-8. doi:10.1371/journal.pone.0000610

12. Maass PG, Luft FC, Bähring S. Long non-coding RNA in health and disease. J Mol Med (2014) 92:337-46. doi:10.1007/s00109-014-1131-8

13. Sonkoly E, Bata-Csörgő Z, Pivarcsi A, Polyánka H, Kenderessy-Szabó A, Molnár $G$, et al. Identification and characterization of a novel, psoriasis susceptibility-related noncoding RNA gene, PRINS. J Biol Chem (2005) 280:24159-67. doi:10.1074/jbc.M501704200

14. Bari L, Bacsa S, Sonkoly E, Bata-Csörgő Z, Kemény L, Dobozy A, et al. Comparison of stress-induced PRINS gene expression in normal human keratinocytes and HaCaT cells. Arch Dermatol Res (2011) 303:745-52. doi:10.1007/ s00403-011-1162-8

15. Yu T-M, Palanisamy K, Sun K-T, Day Y-J, Shu K-H, Wang I-K, et al. RANTES mediates kidney ischemia reperfusion injury through a possible role of HIF$1 \alpha$ and LncRNA PRINS. Sci Rep (2016) 6:18424. doi:10.1038/srep18424

16. Pawar K, Hanisch C, Palma Vera SE, Einspanier R, Sharbati S. Down regulated lncRNA MEG3 eliminates mycobacteria in macrophages via autophagy. Sci Rep (2016) 6:19416. doi:10.1038/srep19416

17. Ouwehand K, Spiekstra SW, Waaijman T, Breetveld M, Scheper RJ, de Gruijl TD, et al. CCL5 and CCL20 mediate immigration of Langerhans cells into the epidermis of full thickness human skin equivalents. Eur J Cell Biol (2012) 91:765-73. doi:10.1016/j.ejcb.2012.06.004

18. de Groot M, Teunissen MBM, Ortonne JP, Lambert JR, Naeyaert JM, Picavet DI, et al. Expression of the chemokine receptor CCR5 in psoriasis and results of a randomized placebo controlled trial with a CCR5 inhibitor. Arch Dermatol Res (2007) 299:305-13. doi:10.1007/s00403-007-0764-7

19. Giustizieri ML, Mascia F, Frezzolini A, De Pità O, Chinni LM, Giannetti A, et al. Keratinocytes from patients with atopic dermatitis and psoriasis show a distinct chemokine production profile in response to $\mathrm{T}$ cellderived cytokines. J Allergy Clin Immunol (2001) 107:871-7. doi:10.1067/ mai.2001.114707

20. Wenzel A, Akbasli E, Gorodkin J. RIsearch: fast RNA-RNA interaction search using a simplified nearest-neighbor energy model. Bioinformatics (2012) 28:2738-46. doi:10.1093/bioinformatics/bts519

21. Smith C, Heyne S, Richter AS, Will S, Backofen R. Freiburg RNA tools: a web server integrating INTARNA, EXPARNA and LOCARNA. Nucleic Acids Res (2010) 38:W373-7. doi:10.1093/nar/gkq316

22. Busch A, Richter AS, Backofen R. IntaRNA: efficient prediction of bacterial sRNA targets incorporating target site accessibility and seed regions. Bioinformatics (2008) 24:2849-56. doi:10.1093/bioinformatics/btn544
23. Wright PR, Georg J, Mann M, Sorescu DA, Richter AS, Lott S, et al. CopraRNA and IntaRNA: predicting small RNA targets, networks and interaction domains. Nucleic Acids Res (2014) 42:W119-23. doi:10.1093/nar/gku359

24. Derrien T, Johnson R, Bussotti G, Tanzer A, Djebali S, Tilgner H, et al. The GENCODE v7 catalog of human long noncoding RNAs: analysis of their gene structure, evolution, and expression. Genome Res (2012) 22:1775-89. doi:10.1101/gr.132159.111

25. Kung JTY, Colognori D, Lee JT. Long noncoding RNAs: past, present, and future. Genetics (2013) 193:651-69. doi:10.1534/genetics.112.146704

26. Heward JA, Lindsay MA. Long non-coding RNAs in the regulation of the immune response. Trends Immunol (2014) 35:408-19. doi:10.1016/j. it.2014.07.005

27. Széll M, Danis J, Bata-Csörgő Z, Kemény L. PRINS, a primate-specific long non-coding RNA, plays a role in the keratinocyte stress response and psoriasis pathogenesis. Pflügers (2016) 468:935-43. doi:10.1007/s00424-016-1803-Z

28. Kopfnagel V, Wittmann M, Werfel T. Human keratinocytes express AIM2 and respond to dsDNA with IL-1 $\beta$ secretion. Exp Dermatol (2011) 20:1027-9. doi:10.1111/j.1600-0625.2011.01382.x

29. Ablasser A, Bauernfeind F, Hartmann G, Latz E, Fitzgerald KA, Hornung V. RIG-I dependent sensing of poly(dA-dT) via the induction of an RNA polymerase III transcribed RNA intermediate. Nat Immunol (2009) 10:1065-72. doi:10.1038/ni.1779

30. Szabó K, Bata-Csörgő Z, Dallos A, Bebes A, Francziszti L, Dobozy A, et al. Regulatory networks contributing to psoriasis susceptibility. Acta Derm Venereol (2014) 94:380-5. doi:10.2340/00015555-1708

31. Wang $\mathrm{S}$, Liu $\mathrm{D}$, Ning $\mathrm{W}, \mathrm{Xu} \mathrm{A}$. Cytosolic dsDNA triggers apoptosis and pro-inflammatory cytokine production in normal human melanocytes. Exp Dermatol (2015) 24:298-300. doi:10.1111/exd.12621

32. Szegedi K, Göblös A, Bacsa S, Antal M, Németh IB, Bata-Csörgő Z, et al. Expression and functional studies on the noncoding RNA, PRINS. Int J Mol Sci (2013) 14:205-25. doi:10.3390/ijms14010205

33. Szcześniak MW, Makałowska I. IncRNA-RNA interactions across the human transcriptome. PLoS One (2016) 11:e0150353. doi:10.1371/journal. pone. 0150353

34. Scheller J, Chalaris A, Schmidt-Arras D, Rose-John S. The pro- and anti-inflammatory properties of the cytokine interleukin-6. Biochim Biophys Acta (2011) 1813:878-88. doi:10.1016/j.bbamcr.2011.01.034

35. Gutschner T, Diederichs S. The hallmarks of cancer: a long non-coding RNA point of view. RNA Biol (2012) 9:703-19. doi:10.4161/rna.20481

36. Bandyopadhyay S, Mitra R. TargetMiner: microRNA target prediction with systematic identification of tissue-specific negative examples. Bioinformatics (2009) 25:2625-31. doi:10.1093/bioinformatics/btp503

37. Kapusta A, Feschotte C. Volatile evolution of long noncoding RNA repertoires: mechanisms and biological implications. Trends Genet (2014) 30:439-52. doi:10.1016/j.tig.2014.08.004

Conflict of Interest Statement: The authors declare that the research was conducted in the absence of any commercial or financial relationships that could be construed as a potential conflict of interest.

Copyright ( 2017 Danis, Göblös, Bata-Csörgö, Kemény and Széll. This is an openaccess article distributed under the terms of the Creative Commons Attribution License (CC BY). The use, distribution or reproduction in other forums is permitted, provided the original author(s) or licensor are credited and that the original publication in this journal is cited, in accordance with accepted academic practice. No use, distribution or reproduction is permitted which does not comply with these terms. 